\title{
Satisfacción de clientes con el servicio de instituciones educativas del sector privado, ubicadas en la Ciudad de Xalapa-Enríquez, México
}

\section{Customer satisfaction with the service of educational institutions in the private sector, located in the city of Xalapa-Enríquez, México}

Judith Guadalupe Montero-Mora; Rosa María Cantón-Croda²

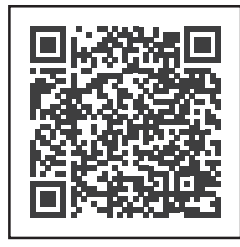

Palabras claves:

satisfacción del cliente, calidad, servicio, educación

Articulo de investigación Fecha de recepción: 23/05/2020

Fecha de aceptación: 20/06/2020

Esta publicación se encuentra bajo licencia:

Creative Commons

Reconocimiento-

NoComercial-

SinObraDerivada 4.0

Internacional

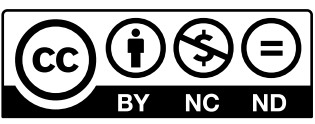

\section{Resumen}

La educación es considerada como un servicio, por lo que toda institución educativa sabe de la importancia de sus clientes y reconoce a la calidad en el servicio y la satisfacción de sus clientes como un factor importante para mantenerse en el actual mercado competitivo. Sin embargo, es importante identificar qué dimensiones y variables de una institución educativa satisfacen las necesidades de los clientes. Por esta razón, el objetivo de la investigación fue identificar las dimensiones y variables que contribuyen en la satisfacción de clientes directos (estudiante) e indirectos (padres) de instituciones educativas privadas, en nivel inicial, preescolar, primaria, secundaria, medio superior, superior y capacitación para el trabajo. El método Delphi se utilizó para el diseño y validación del instrumento, el cual quedó conformado por 22 ítems en escala diferencial semántico con valores de 1 a 7 y seis dimen-

Maestra en Ciencias Administrativas. Candidato a Doctor en Planeación Estratégica y Dirección de Tecnología en UPAEP-Universidad. Universidad Veracruzana (UV), Profesor de Tiempo Completo, México.jmontero@uv.mx, @JGMoMo_24, https://orcid.org/0000-0003-4855-3248

2 Doctora en Ciencias Computacionales con especialidad en Sistemas de Información, ITESM-Campus Ciudad de México. Universidad Popular Autónoma del Estado de Puebla (UPAEP-Universidad), Decana de Posgrados en Ingenierías y Negocios, México, rosamaria.canton@upaep.mx, https:// orcid.org/0000-0002-5469-8964 


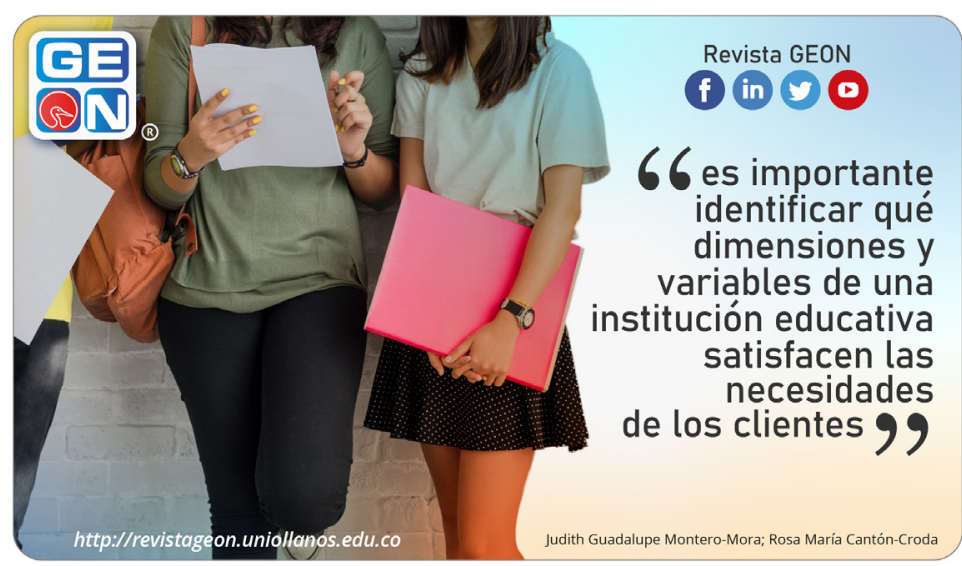

siones: infraestructura, personal docente, servicio administrativo, ambiente, resultados y satisfacción. Para determinar el tamaño de muestra se aplicó un muestreo aleatorio estratificado con asignación proporcional al tamaño del estrato (niveles y escuelas), la muestra fue de 83 escuelas ubicadas en la ciudad de Xalapa-Enríquez, México y 354 clientes. Con un modelo de Ecuaciones Estructurales se encontró que, a excepción de la infraestructura, las cuatro dimensiones restantes mostraron una influencia positiva en la satisfacción, es decir, se encontró un modelo de satisfacción en el que se distinguió el aporte de las dimensiones y de las variables medidas dentro de cada dimensión, sobre la satisfacción de los clientes.

Palabras claves: satisfacción del cliente, calidad, servicio, educación

\section{Abstract}

Education is considered as a service, so that every educational institution knows about the importance of its customers and recognizes the quality of service and customer satisfaction as an important factor to stay in the current competitive market. However, it is important to identify which dimensions and variables of an educational institution meet the needs of customer. For this reasons, the objective of this investigation was to identify the dimensions and variables that contribute to the 
Cómo citar este artículo /

Toreference this article:

Montero Mora, J., \& Cantón Croda, R. (2020). Satisfacción de clientes con el servicio de instituciones educativas del sector privado, ubicadas en la Ciudad de XalapaEnríquez, México. Revista GEON (Gestión, Organizaciones Y

Negocios), 7(2), 1-15. https://doi. org/10.22579/23463910.216 satisfaction of direct (student) and indirect customer (parents) of private educational institutions, at initial, kindergarten, elementary school, middle school, high school, college and job training levels. The Delphi method was used for the design and validation of the instrument, which was made up of 22 items in semantic differential scale with values of 1 to 7 and six dimensions: infrastructure, teaching staff, administrative service, environment, results and satisfaction. For determining the sample size a stratified random sampling was applied with allocation proportional to the size of the stratum (levels and schools), the sample was 83 schools located in the city of Xalapa-Enríquez, México and 354 users. With a structural equations model it was found that, with the exception of infrastructure, the remaining four dimensions showed a positive influence on satisfaction, this is, a satisfaction model was found in which was distinguished the contribution of the dimensions and the variables measured within each dimension, on the customer satisfaction.

Keywords: customer satisfaction, quality, services, education

Código JEL: 129

\section{Introducción}

La educación es considerada como un servicio y además una importante fuente de ingresos para muchos países con economías en desarrollo (Khoo, Ha, y McGregor, 2017). Toda institución educativa sabe de la importancia de sus clientes, por lo que, la satisfacción de estos, es un factor importante para su permanencia en el mercado (Mestrovic, 2017).

Diversos autores han propuesto modelos y escalas para medir calidad en el servicio y satisfacción del cliente, sin embargo, se ha identificado que los principales modelos se diseñaron, en su mayoría, en sectores de servicios diferentes al sector educativo; en contextos internacionales, donde los clientes tienen diferentes expectativas y necesidades. En el caso de modelos de satisfacción desarrollados en el contexto educativo, la medición de la satisfacción se realiza sólo a clientes directos (estudiantes), sin considerar clientes indirectos, como lo es el caso de los padres de los estudiantes; aunado a esto los estudios 
Satisfacción de clientes can el servicia de instituciones educativas del sectar privado, ubicadas en la Ciudad de Xalapa-Enríquez, Méxica

se enfocan principalmente en el nivel superior de educación. Por lo anterior, el objetivo de la investigación fue identificar las dimensiones y variables que contribuyen en la satisfacción de clientes directos (estudiante) e indirectos (padres) de instituciones educativas privadas, en nivel inicial, preescolar, primaria, secundaria, medio superior, superior y capacitación para el trabajo, utilizando un instrumento de medición diseñado y validado para su aplicación en ambos tipos de clientes. La investigación se centra en instituciones pertenecientes a un estrato específico, el sector privado, debido a la importancia que estas han adquirido y al número que actualmente representan, pues en los últimos años, han mostrado un incremento importante. Todas las instituciones incluidas en la investigación aunque son de diferentes niveles, pertenecen al sector privado y tienen validez oficial ante la Secretaría de Educación Pública (SEP) en México. El levantamiento de las encuestas se realizó en instituciones ubicadas en la ciudad de Xalapa, Veracruz, México; en los meses de Enero-Marzo de 2019.

El trabajo considera en un primer apartado el contexto teorico, haciendo énfasis en que los clientes son tanto los estudiantes como los padres de éstos. Un segundo apartado presenta la metodología de dieño muestral aplicada para obtener una muestra representativa de instituciones del sector privado, y la metodología de análisis estadístico que llevó a la propuesta de un modelo de satisfacción del cliente. El tercer apartado presenta los principales resultados y se reali- za una breve discusión de éstos en el apartado final.

\section{Contexto teórico}

Aunque existe un sin número de definiciones sobre el concepto de calidad en el servicio, todas coinciden en que el propósito principal es la satisfacción del cliente (Hernández y Félix, 2010). Por consiguiente, el éxito para alcanzar la calidad en empresas de servicio puede ser medido por el nivel de satisfacción de los clientes con base en el servicio recibido, reflejándose en su regreso (Alén González y Fraiz Brea, 2006) (Valenzuela, BuenteIlo, Gomez, \& Villareal, 2019).

\section{La calidad en el contexto educativo}

Los orígenes de la calidad como ciencia inician en 1920 en el mundo empresarial, enfocándose solo en los productos. Sin embargo, le tomó poco tiempo expandirse al contexto educativo (Tumino y Poitevin, 2013), el cual además de ser dinámico, también es cambiante, con una gran competencia en los sectores público y privado, sobre todo en el nivel superior (Yusoff, McLeay, y Woodruffe-Burton, 2015).

En el cotexto educativo, son diversos los autores que han realizado propuestas de modelos de calidad, Gento Palacios y Montes Castillo (2010); proponen un modelo para toda institución educativa, que considera como principales indicadores de calidad, el producto educativo, la satisfacción de los alumnos, la satisfacción del personal que labora en la institución y el impacto de la educación. 
Para Capelleras y Venciana (2001) si se desea afirmar que una institución de educación tiene un enfoque de calidad es importante definir cuáles son el producto, las actividades y los clientes clave de ésta. Abdullah (2006) refiere que en el contexto educativo estudiantes, académicos, gobierno y familias todos son clientes del sistema educativo, considerando a los estudiantes como los "clientes primarios". Tanto Gento Palacios y Montes Castillo (2010) y Abdullah (2006) coiciden en la satisfacción del alumno como parte de los resultados clave de una institución educativa, sin embargo, como lo señala (Abdullah, 2006), las familas de estudiantes son también clientes, por lo que la opinión de éstos acerca de la institución es trascendente.

Dentro de los modelos que se consideran parte de la teoría fundamental en el área de la calidad del servicio y satisfacción del cliente y además han sido base para diversas investigaciones en el sector educativo se encuentra, el modelo SERVQUAL de (Parasuraman, Zeithamly Berry, 1985), uno de los más utilizados actualmente en diversos sectores de servicio. El Modelo SERVPERF de Cronin y Taylor (Cronin y Taylor, 1992). Sin embargo, aunque son los modelos que han servido como base en diferentes investigaciones, se considera que para el sector educativo estos requieren de diversas modificaciones para captar información acorde a este sector.

Con el anterior contexto y apoyando lo señalado por Tumino y Poitevin (2013) las escalas de percepción de los servi- cios deben estar contextualizadas culturas y experiencias de la población bajo estudio, por lo que se considera que si se desea medir la satisfacción de clientes (Torres-Flórez, 2019) de instituciones educativas de un sector educativo especifico, el privado, debe considerarse un modelo con variables acordes a este sector, aunado a esto es de interés también conocer la percepción no sólo de los clientes directos (estudiantes), sino también de clientes indirectos (padres/tutores) en los diferentes niveles de educación; ya que se se considera que que nó sólo el estudiante es cliente de una institución educativa, los son también los padres quienes, en su caso, suelen aportar el recurso económico para la educación de los hijos.

\section{Materiales y métodos}

La investigación es de tipo cuantitativo ya que se realizó la recolección de datos mediante la aplicación de un cuestionario a una muestra probabilística de clientes directos e indirectos de instituciones de educación privada y posteriormente se realizó un análisis estadístico utilizando un Modelo de Ecuaciones Estructurales. El tipo de diseño fue observacional pues nuestro interés era medir a través de la aplicación de un cuestionario, la percepción de la calidad en el servicio y por consiguiente el nivel de satisfacción de clientes. Fue transversal porque la medición se hizo en un solo momento temporal. El alcance fue descriptivo ya que se buscaba especificar las dimensiones y variables que definen la satisfacción de clientes en instituciones de educación en el 
Satisfacción de clientes can el servicio de instituciones educativas del sector privado, ubicadas en la Ciudad de Xalapa-Enríquez, México

sector privado. (Hernández, Fernández, y Baptista, 2014).

\section{Selección de la muestra}

Dado que el objetivo fue obtener una muestra representativa del sector privado, la población de interés fueron $N=5,145$ clientes de 622 instituciones educativas de dicho sector; el alumnado, en el caso de bachillerato, nivel superior y capacitación para el trabajo; y en los niveles básicos, inicial, preescolar, primaria y secundaria los padres y madres de familia. En la determinación del tamaño de muestra se utilizó un muestreo en dos etapas. En la primera etapa, se consideró el tipo de nivel como estrato (siete estratos) y mediante un muestreo aleatorio estratificado se determinó un tamaño de muestra global de $n=83$ instituciones, esta muestra fue distribuida dentro de cada uno de los siete niveles utilizando asignación proporcional al tamaño de cada estrato, resultando 4 instituciones de inicial, 13 de preescolar, 9 de primaria, 6 de secundaria, 13 de educación media superior, 13 de superior y 25 de capacitación para el trabajo. La segunda etapa considero la determinación del tamaño de muestra dentro de cada escuela, se realizó a través de un muestreo aleatorio estratificado con asignación proporcional al tamaño del estrato, considerando ahora como estratos a cada una de las 83 instituciones y la población de $N=5,145$ alumnos. Se determinó un tamaño de muestra $n=358$ clientes, este se asignó proporcionalmente dentro de cada escuela. La selección de clientes en cada escuela se realizó de manera aleatoria con la finalidad de eliminar los posibles sesgos de selección y asegurar que la muestra representaba apropiadamente a toda la población. En la determinación de las muestras se utilizo un nivel de confiabilidad del 95\%, un margen de error del $10 \%$ y se asumió máxima varianza (L. Scheaffer Richard, Mendenhall WiIliam, 2000):

\section{Diseño y validación del instrumento}

Para el diseño y validación del instrumento se recurrió al método Delphi por consulta a expertos, (López-Gómez, 2018) y (García-Ruiz y Lena-Acebo, 2018). Se contó con el apoyo de 12 expertos en el área de calidad y servicio al cliente (Skulmoski y Hartman, 2007). Se realizó un análisis de validez de contenido del instrumento con el Coeficiente $V$ de Aiken (Aiken, 1980) se consideró un valor aceptable para $V=0.70$. La versión final del instrumento incluyó 22 ítems en una escala diferencial semántico con valores de 1 a 7 agrupados en seis dimensiones: 1) infraestructura; 2) personal docente; 3) servicios administrativos; 4) ambiente; 5) resultados y 6) satisfacción; y dos preguntas abiertas. Ese instrumento se aplicó a una muestra piloto de 50 clientes. Con esta muestra se observó un valor del estadístico Alfa de Cronbach de 0.98, indicando una buena consistencia interna del instrumento (Cronbach, 1951).

\section{Análisis estadístico}

Para realizar un análisis descriptivo de la valoración general de los 
encuestados, se generó una nueva variable que se llamó "satisfacción global" a partir de la suma de los puntajes de los 22 ítems, así el puntaje mayor que se podría observar en dicha variable es 154 y el puntaje menor 22. Con este puntaje se calcularon estadísticas descriptivas como la media y la desviación estándar y se realizó un histograma para describir la distribución de la satisfacción global. Para preguntas abiertas se realizó un análisis textual. El análisis que permitió llegar a la propuesta de un modelo de satisfacción de clientes de instituciones educativas privadas fue un Modelo de Ecuaciones Estructurales (Hair Jr, Black, Babin, y Anderson, 2014). Los análisis fueron ejecutados en el lenguaje de programación R ( R Core Team, 2018).

\section{Resultados}

Del total de encuestados, el 59\% fueron estudiantes y $41 \%$ fueron padres o tutores de estudiantes en nivel inicial, preescolar, primaria y secundaria. Por género el mayor número de clientes encuestados fueron del femenino con un $61.4 \%$ y $38.6 \%$ del masculino. La Figura 1 muestra la distribución del puntaje de satisfacción global (el puntaje mínimo que se podría obtener es 22 y el máximo 154), puede observarse que 343 de los puntajes (82.06\%) se encuentra por arriba de los 100 puntos, la media de la satisfacción global fue de 130.39. Esta valoración global, puede considerarse como un indicador de que los encuestados se encontraban satisfechos con el servicio de las instituciones incluidas en la muestra.
Figura 1. Distribución del puntaje de Satisfacción global en escuelas del sector privado

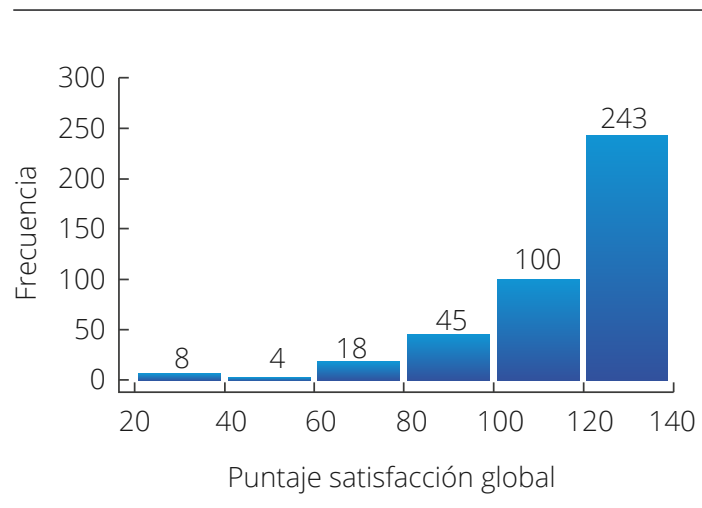

Fuente: Elaboración propia, 2019

Figura 2. Características que distinguen a las instituciones del sector privado

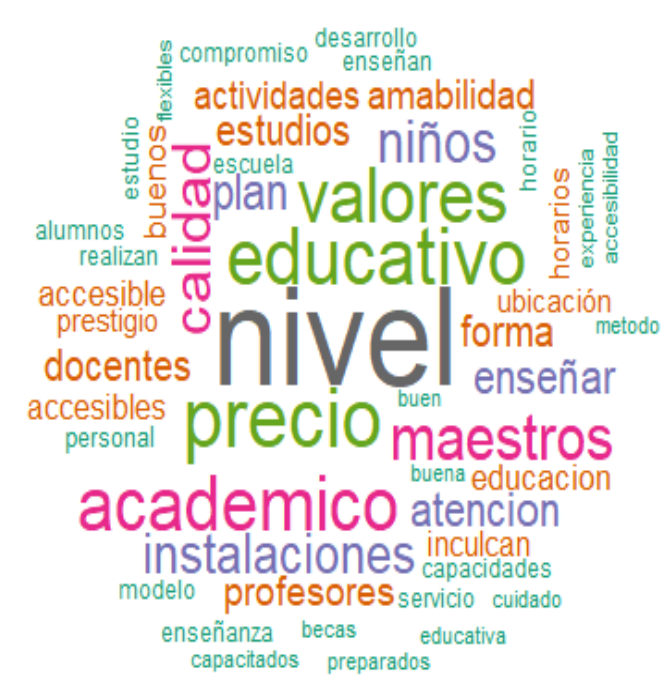

Fuente: Elaboración propia, 2019

El análisis que se realizó para la pregunta relacionada con lo que los clientes consideraban distinguía a la institución que evaluaron de otras instituciones que ofrecían el mismo servicio, se muestra en la Figura 2, que "el nivel educativo (académico), es considerado como algo distintivo, destacan 
también los valores que enseña la institución. El precio resultó ser también un factor que distingue a la institución evaluada, pues los clientes entrevistados indicaron que los precios (en las colegiaturas) eran accesibles.

El modelo estructural propuesto se muestra en la Figura 3. Las líneas rectas denotan las relaciones de regresión entre las dimensiones, cada línea recta tiene un parámetro a estimar, por ejemplo: $P_{S A T, P D}$ es el parámetro que se estima entre la relación de la dimensión satisfacción (SAT) y personal docente $(P D)$. Las líneas curvas denotan las covarianzas entre las dimensiones. Las variables $X_{1}$ a $X_{20} y Y_{1}$, $Y_{2}$ denotan las variables observadas.
Las hipótesis que se plantean en el modelo de la Figura 5, asociadas a cada dimensión y al impacto que tienen en la satisfacción de los usuarios son:

1) La percepción de la infraestructura está relacionada positivamente con la satisfacción de los clientes: $H_{1}: I N F \rightarrow S A T$, 2) La percepción sobre el Personal docente está relacionada positivamente con la satisfacción de los clientes: $H_{2}: P D \rightarrow S A T$, 3) La percepción de los servicios administrativos está relacionada positivamente con la satisfacción de los clientes: $H_{3}: S A \rightarrow S A T$, 4) La percepción sobre el ambiente institucional está relacionada positivamente

Figura 3. Modelo estructural (modelo de medida)

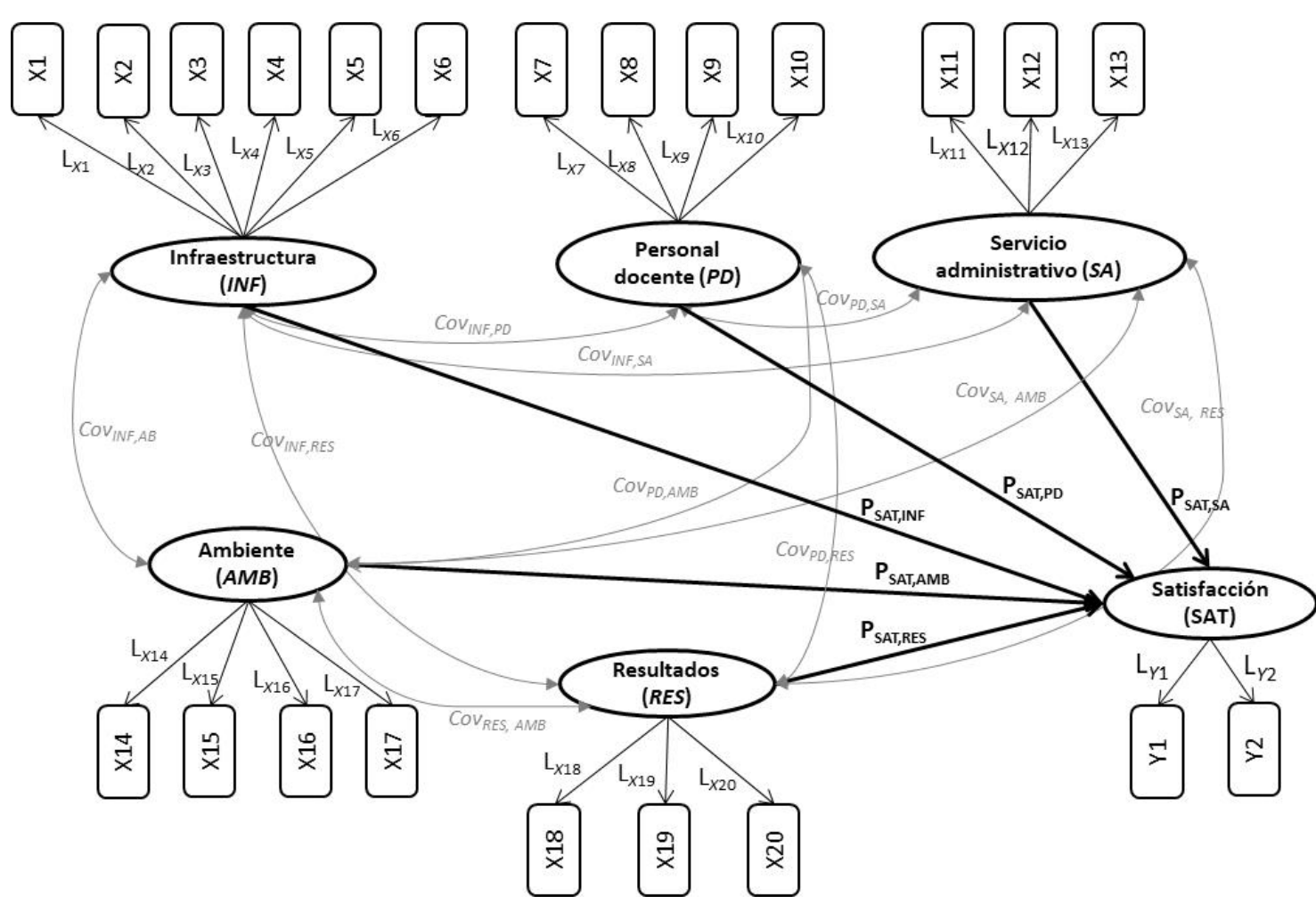

Fuente: Elaboración propia, 2019 
con la satisfacción de los clientes: $H_{4}: A M B \rightarrow S A T$, 5) La percepción sobre los resultados de la institución están relacionados positivamente con la satisfacción de los clientes : $\mathrm{H}_{5}: R E S \rightarrow S A T$

En la evaluación del ajuste del modelo de ecuaciones estructurales, se considera un ajuste aceptable si el valor del estadístico ji-cuadrado dividido por su grados de libertad ( $\left.\chi^{2} / g . l\right)$ se encuentra entre 2 y 3 y como límite un valor de 5 (Escobedo, Hernández, Estebané, y Martínez, 2016), el valor observado es 3.94 por esto se considera que el modelo de satisfacción propuesto tiene un ajuste aceptable. El valor de CFI (índice de ajuste comparativo) es 0.94, indica que al menos el 94\% de la covarianza en los datos es reproducida por el modelo; TLI (índice de Tucker-Lewis) es de 0.93, ambos índices se consideran aceptables ya que son mayores al valor de referencia 0.92 (Hair Jr et al., 2014), indicando un buen ajuste del modelo. El RMSEA representa que tan bien se ajusta un modelo a una población, es aceptable para valores menores a 0.8, el valor observado es de 0.087, con un intervalo de confianza de $(0.81,0.094)$, por lo que se considera aceptable. Finalmente SRMR (Raíz del residuo cuadrático promedio estandarizado), mide las varianzas y covarianzas de la muestra y si éstas difieren de las estimaciones obtenidas, valores cercanos a 0 indican un ajuste casi perfecto (Escobedo et al., 2016), el valor observado es de 0.041, por lo que se considera aceptable. En conclusión, el modelo tiene un buen ajuste.
En relación a los parámetros estimados, debe identificarse si son estadísticamente significativos. Los parámetros del modelo de medida, indican la carga factorial de las variables observadas en la dimensión a la que corresponden. En la Figura 3 están denotados para las variales exógenas por LX1 a LX20, y para las variables endógenas por LY1 y LY2. El análisis arrojó que todos los parámetros son significativos, $(p<0.05)$ (Tabla I).

Tabla I. Parámetros estimados por cada relación en el modelo de medida

\begin{tabular}{|c|c|c|c|}
\hline $\begin{array}{c}\text { Relación en } \\
\text { el modelo de } \\
\text { medida }\end{array}$ & $\begin{array}{l}\text { Parámetro } \\
\text { estimado }\end{array}$ & $\begin{array}{l}\text { Error } \\
\text { estándar }\end{array}$ & Valor- $p$ \\
\hline $\mathrm{L}_{x_{1}} \rightarrow \mathrm{NF}$ & 0.768 & & \\
\hline $\mathrm{L}_{x_{2}} \rightarrow \mathrm{NF}$ & 0.799 & 0.063 & 0.000 \\
\hline $\mathrm{L}_{x_{3}} \rightarrow \mathrm{NF}$ & 0.849 & 0.068 & 0.000 \\
\hline $\mathrm{L}_{x_{4}} \rightarrow$ INF & 0.766 & 0.069 & 0.000 \\
\hline $\mathrm{L}_{x 5} \rightarrow \mid \mathrm{NF}$ & 0.818 & 0.063 & 0.000 \\
\hline $\mathrm{L}_{x_{6}} \rightarrow \mid \mathrm{NF}$ & 0.449 & 0.101 & 0.000 \\
\hline $\mathrm{L}_{X 7} \rightarrow \mathrm{PD}$ & 0.892 & & \\
\hline $\mathrm{L}_{x 8} \rightarrow \mathrm{PD}$ & 0.937 & 0.036 & 0.000 \\
\hline $\mathrm{L}_{x 9} \rightarrow \mathrm{PD}$ & 0.927 & 0.037 & 0.000 \\
\hline $\mathrm{L}_{x 10} \rightarrow \mathrm{PD}$ & 0.862 & 0.042 & 0.000 \\
\hline $\mathrm{L}_{x 11} \rightarrow \mathrm{SA}$ & 0.820 & & \\
\hline $\mathrm{L}_{x 12} \rightarrow \mathrm{SA}$ & 0.925 & 0.042 & 0.000 \\
\hline $\mathrm{L}_{x 13} \rightarrow \mathrm{SA}$ & 0.935 & 0.041 & 0.000 \\
\hline$L_{x 14} \rightarrow A M B$ & 0.894 & & \\
\hline $\mathrm{L}_{x 15} \rightarrow \mathrm{AMB}$ & 0.891 & 0.040 & 0.000 \\
\hline $\mathrm{L}_{x 16} \rightarrow \mathrm{AMB}$ & 0.896 & 0.041 & 0.000 \\
\hline $\mathrm{L}_{x 17} \rightarrow \mathrm{AMB}$ & 0.869 & 0.046 & 0.000 \\
\hline $\mathrm{L}_{x 18} \rightarrow \mathrm{RES}$ & 0.960 & & \\
\hline $\mathrm{L}_{\chi 19} \rightarrow \mathrm{RES}$ & 0.969 & 0.022 & 0.000 \\
\hline $\mathrm{L}_{X 20} \rightarrow \mathrm{RES}$ & 0.841 & 0.033 & 0.000 \\
\hline $\mathrm{L}_{\gamma 1} \rightarrow \mathrm{SAT}$ & 0.883 & & \\
\hline $\mathrm{L}_{12} \rightarrow \mathrm{SAT}$ & 0.895 & 0.038 & 0.000 \\
\hline
\end{tabular}

Fuente: Elaboración propia, 2019 
Los parámetros estimados de la Tabla I muestran que uno de los constructos mejor explicados es el de Resultados, pues las cargas son las mayores, las dos cargas que destacan de este constructo son $L_{x 18}=0.960$ y $L_{x 19}=$ 0.969 y corresponden a la variables observadas X18: la institución estimula (frena) el desarrollo de las capacidades (poder hacer) del alumno y X19: la institución estimula (frena) el desarrollo de habilidades (saber hacer) del alumno. Sin embargo, los constructos Personal docente y Servicio administrativo tienen cargas factoriales que indican también que estos constructos están explicados por sus correspondientes variables. Para el constructo Ambiente las cuatros variables que la explican aportan información parecida, pues las cargas oscilan entre 0.869 y 0.891. En el constructo Satisfacción, las dos variables que la conforman aportan también información similar, las cargas son: $L_{\gamma_{1}}=0.883$ y $L_{\gamma_{2}}=0.895$. Finalmente, el constructo Infraestructura es en el que se observa las cargas menores, es decir, es el menos explicado por sus variables, se presenta en este la carga más pequeña $L_{x 6}=0.449$ correspondiente a X6: las adecuaciones de la institución para la movilidad de personas con capacidades diferentes son apropiadas (inapropiadas).

Ahora se analizan los parámetros de las relaciones entre las dimensiones, en la Tabla II se presentan los parámetros estimados del modelo propuesto de satisfacción. Puede observarse que, a excepción de la infraestructura, todos los parámetros son significativos $(p<0.05)$ y en la dirección propuesta en las hipótesis. Sin embargo, en general, dado que cuatro de las cinco estimaciones son consistentes con las hipótesis, se considera que los resultados apoyan el modelo propuesto. De los parámetros estimados se interpreta lo siguiente: $P_{p}$. $D_{D S A T}=0.154$ es el valor con el que la dimensión Personal Docente $(P D)$ influye sobre la dimensión Satisfacción (SAT), siendo esta la de menor impacto. La dimensión con mayor impacto en la Satisfacción, es la de Resultados (RES) con un valor $\mathrm{P}_{\text {RESSSAT }}=0.371$,

Tabla II. Parámetros estimados por cada relación en el modelo estructural

\section{Relación estructural}

1) $H_{1}:$ INF $\rightarrow S A T$

2) $H_{2}: P D \rightarrow S A T$

3) $H_{3}: S A \rightarrow S A T$

4) $H_{4}: A M B \rightarrow S A T$

5) $H_{5}: R E S \rightarrow S A T$
Parámetro estimado

Error estándar

Valor- $p$

0.409

0.305

0.235

0.262

0.449

0.007

Fuente: Elaboración propia, 2019 
seguida del Ambiente $(A M B)$ con un valor de $P_{A M B, S A T}=0.290$ y servicio administrativo (SA) con un valor de $P_{\text {SA,SAT }}$ = 0.185. La dimensión Infraestructura es no significativa, por lo tanto puede interpretarse que esta dimensión no tiene impacto en la satisfacción.

La Figura 4 muestra el modelo estructural para la satisfacción de clientes de instituciones de educación privada con los parámetros estimados.

\section{Discusión}

Se encontró que de manera general, las instituciones incluidas en la muestra fueron evaluadas con puntajes ubicados en un nivel alto. El precio (costo de las colegiaturas), lo valores que enseña la institución, el nivel académico (educativo) entre otras, son consideradas como variables importantes en la satisfacción de los clientes para una evaluación positiva de la institución.

Los hallazgos del modelo de ecuaciones estructurales permitieron identificar las variables que dentro de cada dimensión tuvieron un mayor peso. Así, en la dimensión Resultados fueron la institución estimula (frena) el desarrollo de capacidades (poder hacer) del alumno y la institución estimula (frena) el desarrollo de habilidades (saber hacer) del alumno. Para personal docente las variables el personal docentes es competente (incompetente) y el personal docentes está actualizado (desactualizado). En la dimensión de servicio administrativo, el personal administrativo es amable (descortés) y el personal

Figura 4 Modelo estructural estimado para la satisfacción de clientes de instituciones educativas del sector privado

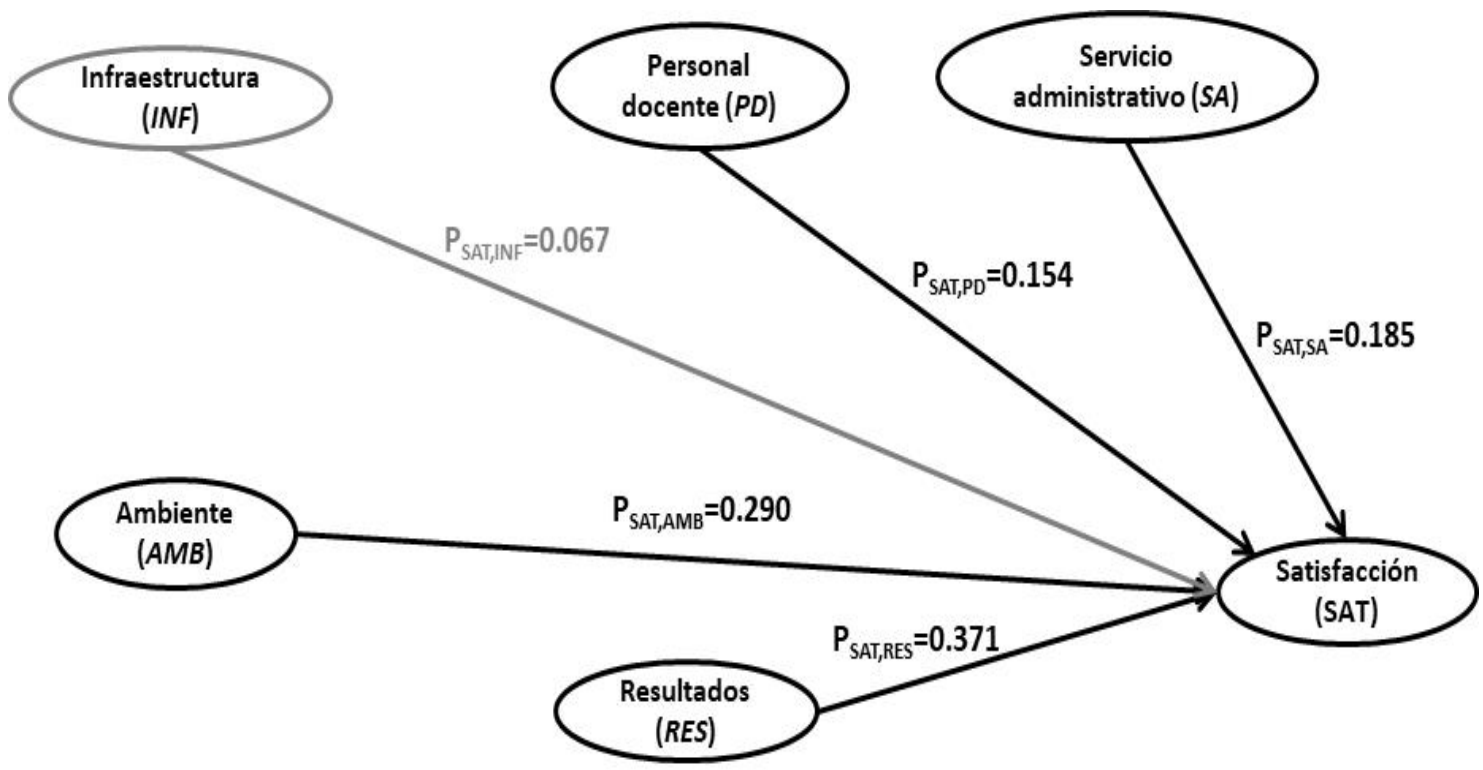

Fuente: Elaboración propia, 2019 
Satisfacción de clientes can el servicio de instituciones educativas del sector privado, ubicadas en la Ciudad de Xalapa-Enríquez, México

administrativo es eficaz (ineficaz) fueron las variable de mayor peso. Para la dimensión ambiente, las cuatros variables que la explican aportan información parecida, el ambiente en la institución es favorable (desfavorable) para la buena convivencia, la comunicación en la institución es efectiva (ineficiente), los medios de comunicación para el intercambio de información en la institución son eficientes (ineficientes) y en caso de conflicto, las soluciones propuestas por la institución son aceradas (equivocadas). Finalmente para la Infraestructura, el equipamiento de las instalaciones de la institución es moderno (obsoleto) fue la de mayor peso.

A nivel dimensión, los hallazgos de esta investigación pueden compararse con lo encontrado por Capelleras y Venciana (2001), las dimensiones que estos autores consideran conforman la percepción de la calidad de la enseñanza fueron: 1) actitudes y comportamiento del profesorado, 2) competencia del profesorado, 3) contenido del plan de estudios de la carrera, 4) instalaciones y equipamiento, 5) organización de la enseñanza. Las dimensiones de coincidencia con esta investigación puede considerarse son: competencia del profesorado e instalaciones y equipamiento.

La investigación de Tumino y Poitevin (2013) evalúa la calidad de los servicios a partir de las dimensiones 1) competencia del cuerpo docente, 2) organización de la enseñanza o de la carrera, 3) comunicación y clima organizacional, 4) actitudes y comportamiento del cuerpo docente, 5) instalaciones y equipamiento, 6) evaluaciones, 7) asignaturas optativas y 8) efectividad de los procesos administrativos. Con esta investigación las dimensiones de coincidencia puede considerarse son: competencia del cuerpo docente, comunicación y clima organizacional e instalaciones y equipamiento.

Para Álvarez Botello et al. (2015) las dimensiones en la satisfacción de los estudiantes son: 1) plan de estudios, 2) capacitación y habilidad para la enseñanza de los docentes, 3) métodos de enseñanza y evaluación, 4) nivel de autorrealización del estudiante, 4) servicios de apoyo, 5) servicios administrativos, 6) ambiente propicio, 7) infraestructura. Las dimensiones de coincidencia con estos autores puede considerarse que son capacitación y habilidad para la enseñanza de los docentes, servicios administrativos, ambiente propicio e infraestructura.

Alvarado-Lagunas et al. (2015) las dimensiones que influyen en la percepción de la calidad educativa en orden de importancia (mayor a menor) son 1) desarrollo integral, 2) componente físico, 3) cualificación de la planta docente y 4) habilidad docente. Con esta investigación se considera que se coincide con el componente físico (instalaciones, equipo de cómputo, aire acondicionado, iluminación, etc.) y cualificación de la planta docente. Sin embargo, en la investigación de estos autores el componente físico tiene mayor peso que la planta docente, en la presente investigación la infraestructura no tiene impacto en la satisfacción. 
Es importante señalar que se ajustó un modelo incluyendo también el tipo de cliente entrevistado (estudiante, padre y/o tutor), esto con la finalidad de poder identificar si las variables que explican la satisfacción cambian en función estos, lo que implicaría tener dos modelos, sin embargo, al ajustar el modelo, no se observaron medidas de bondad de ajuste aceptables al incluir esta variable, por lo que se concluyó que el modelo propuesto para la satisfacción de clientes de instituciones de educación del sector privado, incluye personal docente, servicio administrativo, ambiente, resultados y satisfacción, sin hacer distinción en función del cliente encuestado.

\section{Conclusiones}

El modelo que se propone para medir la satisfacción de clientes de instituciones de educación del sector privado, es un buen modelo, destacando que la satisfacción de estos clientes está influenciada de manera positiva por variables como los resultados que muestran las instituciones, el ambiente al interior de estas, el servicio administrativo que opera y el personal docente que labora en ellas. La infraestructura es una variable que no resulta tener un peso importante para explicar la satisfacción de estos clientes.

Existen coincidencias con otras investigaciones y las coincidencias se dan en dimensiones como planta docente, infraestructura, servicio administrativo, ambiente de la institución, sin embargo, debe destacarse que las investigaciones encontradas son en su mayoría en instituciones de educación superior y realizadas sólo a estudiantes, la presente investigación incluye la percepción tanto de estudiantes, como de padres y/o tutores, los clientes indirectos, de ahí que las percepciones de los clientes indirectos, no incluyen dimensiones como evaluaciones, autorrealización del estudiante, desarrollo integral.

Sin embargo, puede considerarse que el aporte más destacado del modelo al que finalmente se llega, consiste en la distinción del aporte de los efectos de las diferentes dimensiones sobre la satisfacción de los clientes. Si bien, cada una de las dimensiones consideradas en el análisis ejerce un efecto significativo y positivo sobre la satisfacción de los clientes (a excepción de la infraestructura), el modo en que lo hacen no es igual; así como tampoco es igual la importancia que tienen las diferentes variables (ítems) medidas dentro de cada dimensión en la valoración global de la misma.

Por lo tanto se considera que se ha construido un modelo de la satisfacción de clientes de instituciones de educación del sector privado desde dos planos diferentes: uno global en el que se distingue qué dimensiones inciden en el aumento de la satisfacción de los clientes y un plano más particular en el que dentro de cada dimensión se identifcan las variables responsables de la valoración de los clientes. Dados los hallazgos, se deja abierta la posibilidad de que en una futura investigación con características similares se incluya en el modelo de satisfacción una dimensión 
Satisfacción de clientes can el servicia de instituciones educativas del sectar privado, ubicadas en la Ciudad de Xalapa-Enríquez, Méxica

relacionada con el precio. Asi mismo, dado que el interés de la presente investigación fue especificamente el sector privado, se deja abierta la posibilidad de que en futuras invstigaciones se consideren varibles como los diferentes niveles.

\section{Referencias}

Abdullah, F. (2006). The development of HEdPERF: a new measuring instrument of service quality for the higher education sector. International Journal of Consumer Studies, 30(6), 569581. https://doi.org/10.1111/j.14706431.2005.00480.x

Aiken, L. R. (1980). Content validity and reliability of single items or questionnaires. Educational and Psychological Measurement, 40(4), 95-959. https://doi.org/https://doi. org/10.1177/001316448004000419

Alén González, M. E., y Fraiz Brea, J. A. (2006). Relación entre la calidad de servicio y la satisfacción del consumidor. Su evaluación en el ámbito del turismo formal. Investigaciones Europeas de Dirección y Economía de la Empresa vol. 12, 251-272.

Capelleras, J. L., y Venciana, J. M. (2001). Calidad de servicio en la enseñanza universitaria: desarrollo y validación de una escala de medida. Document de treball, (4), 1-36. Recuperado de http://www.uab.cat/servlet/ BlobServer?blobtable=Document\&blobcol=urldocument\&blobheader=application/pdf\&blobkey=id\&blobwhere=1345650483792\&blobnocache=true

Cronbach, L. J. (1951). Coefficient alpha and the internal strucuture of tests. Psychometrika, 16, 297-334.
Cronin, J. J., y Taylor, S. A. (1992). Measuring Service Quality: A Reexamination and Extension. Journal of Marketing, 56(3), 55. https://doi.org/10.2307/1252296

Escobedo, M., Hernández, J., Estebané, V., y Martínez, G. (2016). Modelos de ecuaciones estructurales: Características, fases, construcción, aplicación y resultados. Ciencia \& trabajo, 18(55), 16-22. https://doi.org/10.4067/ S0718-24492016000100004

García-Ruiz, M. E., y Lena-Acebo, F. J. (2018). Aplicación del Método Delphi en el diseño de una investigación cuantitativa sobre el fenómeno FABLAB. Revista de Metodología de Ciencias Sociales. EMPIRIA. No. 40, 129-166.

Gento Palacios, S., y Montes Castillo, M. (8-12, Febrero, 2010). Cómo Elaborar un Diagnóstico de la Calidad de un Centro Educativo: Implementación y Resultados de un Modelo. VII Congreso Internacional, "La Universidad por un Mundo Mejor", La Habana, Cuba, (Vol. 5, pp. 265-288).

Hair Jr, J. F., Black, W. C., Babin, B. J., y Anderson, R. E. (2014). Multivariate Data Analysis. (Pearson, Ed.) (Séptima).

Hernández, R., Fernández, C., y Baptista, P. (2014). Metodología de la investigación. Journal of Chemical Information and Modeling (Vol. 53). https://doi.org/10.1017/ CBO9781107415324.004

Khoo, S., Ha, H., y McGregor, S. L. T. (2017). Service quality and student/customer satisfaction in the private tertiary education sector in Singapore. International Journal of Educational Management, 31(4), 430-444. https://doi. org/10.1108/IJEM-09-2015-0121 
L. Scheaffer Richard, Mendenhall William, O. L. (2000). Elementos de muestreo. (G. E. Iberoamerica, Ed.).

López-Gómez, E. (2018). El método Delphi en la investigación actual en educación: una revisión teórica y metodológica. Educación XX1, 21(1), 17-40. https:// doi.org/10.5944/educXX1.15536

Mestrovic, D. (2017). Service Quality, Students' Satisfaction and Behavioural Intentions in STEM and IC Higher Education Institutions. Interdisciplinary Description of Complex Systems, 15(1), 66-77. https://doi.org/10.7906/ indecs.15.1.5

Parasuraman, A., Zeithaml, V. A., y Berry, L. L. (1985). Model Service Its Quality and Implications for Future. Research Paper, 49(4), 41-50. https://doi. org/10.1016/S0148-2963(99)00084-3

R Core Team. (2018). R: A language and environment for statistical computing. Vienna, Austria. Recuperado de https://www.r-project.org/

Skulmoski, G. J., y Hartman, F. T. (2007). The Delphi Method for Graduate Research. Journal of Information Technology Education, 6(1), 1-21. https://doi. org/10.1.1.151.8144
Torres-Flórez, D. (2019). Estrategia de compensaciones como herramienta de satisfacción laboral. Revista GEON (Gestión, Organizaciones Y Negocios), 6(2), 4-9. https://doi. org/10.22579/23463910.181

Tumino, M. C., y Poitevin, E. R. (2013). Evaluación de la calidad de servicio universitario desde la percepción de estudiantes y docentes: caso de estudio Quality assessment of university service from students and teacher's perceptions: a case study. REICE. Revista Iberoamericana sobre Calidad, 12(2), 63-84. Recuperado de www.rinace. net/reice/

Valenzuela Salazar, N., Buentello Martínez, C., Gomez, L., \& Villareal Sánchez, V. (2019). La atención al cliente, el servicio, el producto y el precio como variables determinantes de la satisfacción del cliente en una pyme de servicios. Revista GEON (Gestión, Organizaciones Y Negocios), 6(2), 18-24. https:// doi.org/10.22579/23463910.159

Yusoff, M., McLeay, F., \& Woodruffe-Burton, H. (2015). Dimensions driving business student satisfaction in higher education. Quality Assurance in Education, 23(1), 86-104. https://doi. org/10.1108/QAE-08-2013-0035 